Z Herz- Thorax- Gefäßchir 2019 · 33:2

https://doi.org/10.1007/s00398-018-0291-1

(c) Springer Medizin Verlag $\mathrm{GmbH}$, ein Teil von Springer Nature 2018

CrossMark

\author{
H. E. Ulmer \\ Heidelberg, Deutschland
}

\title{
Zukunft braucht Herkunft
}

Angeborene Herzfehler bei Kindern gibt es, seit Menschen diesen Planeten bevölkern. Dennoch sind die Versuche, diese Anomalien $\mathrm{zu}$ verstehen, noch nicht mehr als 3 bis 4 Jahrhunderte alt. Aber wozu dient es heute, da die pathologischen Strukturen und die sich daraus ergebenden krank machenden Mechanismen weitgehend aufgeklärt und klassifiziert sind, sich für die Geschichte dieser Entwicklung und der damit verbundenen Menschen $\mathrm{zu}$ interessieren? Heute, da die Herz-Lungen-Maschine in weiten Teilen der Welt der Welt verfügbar ist, macht es da einen Sinn, sich zu erinnern, dass angeborene Herzfehler auch schon zu Beginn des 20. Jh. operativ behandelt wurden, wenn auch mit anderen Möglichkeiten und Ergebnissen?

Ebenso essenziell wie das individuelle Gedächtnis für den einzelnen Menschen, erscheint auch das kollektive Gedächtnis bestimmter Gruppen von Menschen, die sich einer gemeinsamen Aufgabe gewidmet haben oder sich in $\mathrm{Zu}$ kunft widmen werden. Die Empfindung für einen historischen Ablauf sollte sich jedoch nicht alleine in einer chronologischen Aufzählung von Ereignissen erschöpfen. Geschichte sollte für die Erfahrung empfindsam machen, wie bedeutsam es sein kann, dass z. B. bestimmte Dinge vor anderen geschehen, und dass einzelne Ereignisse an einem bestimmten Ort stattfinden und nicht an einem beliebigen anderen. Das Wissen um eine derartige Spur, erworben durch eine historische Analyse von Ideen und Ereignissen, kann zur Entwicklung eigener Bescheidenheit, zu einer Erweiterung des Horizonts und zu einer produktiven Aktivität führen.

Ein solches Interesse stellt das Charakteristikum eines Arztes dar, der mehr als ein reiner Mediziner ist, eines Arztes, der eine Erziehung erfahren und nicht nur eine Ausbildung durchlaufen hat, eines Arztes, der mit den ihm anvertrauten $\mathrm{Pa}$ tienten verbunden und bereit ist, nicht nur die fachliche medizinische Verantwortung für sie zu tragen.

Die Vergangenheit ist nicht im Besitz von Historikern, sondern sie stellt das Eigentum aller Menschen dar. Sie gehört jedem, der sich ihrer bewusst ist, und sie wächst, indem sie geteilt wird.

Prof. Dr. Herbert E. Ulmer Universitätsklinikum Heidelberg

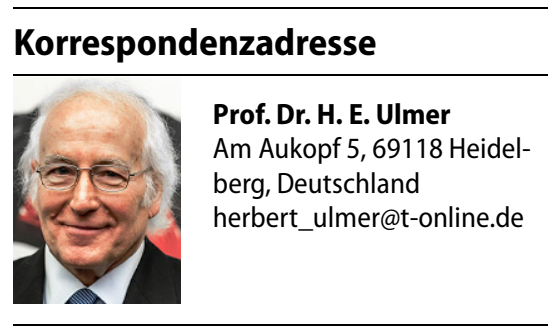

Interessenkonflikt. H.E. Ulmer gibt an, dass kein Interessenkonflikt besteht. 